\title{
Design and Synthesis of Sustain-Acting Melatonin Prodrugs
}

\author{
Phạm Văn Thoại and Nguyen Hai Nam \\ Department of Pharmaceutical Chemistry, Hanoi University of Pharmacy, 13-15 Le Thanh Tong, Hanoi, Vietnam
}

Correspondence should be addressed to Nguyen Hai Nam; nhnam@hotmail.com

Received 19 May 2013; Revised 29 July 2013; Accepted 1 August 2013

Academic Editor: David Bongiorno

Copyright (C) 2013 P. Văn Thoại and N. H. Nam. This is an open access article distributed under the Creative Commons Attribution License, which permits unrestricted use, distribution, and reproduction in any medium, provided the original work is properly cited.

Twelve melatonin amide prodrugs aiming at prolonging the action of melatonin in vivo by improving its half-life were designed and synthesized. Using an $80 \%$ human plasma model, it was found that the aliphatic amide derivatives were relatively stable and melatonin release from these compounds was not sufficient with melatonin release percentage. After 4-hour incubation with $80 \%$ human plasma, the melatonin release percentage achieved only approximately less than $20 \%$. In contrast, the $N^{1}$-succinyl and $N^{1}$ glutaroylmelatonin derivatives (compounds 11 and 12, resp.) were found to release melatonin in much higher rates. After 3-hour incubation in $80 \%$ human plasma, the melatonin release rates from $\mathbf{1 1}$ and $\mathbf{1 2}$ were found to be 67.3 and $75.6 \%$, respectively. From these results, the $N^{1}$-succinyl and $N^{1}$-glutaroylmelatonin derivatives (compounds 11 and 12) could be promising as sustained release prodrugs of melatonin.

\section{Introduction}

Melatonin ( $N$-acetyl-5-methoxytryptamine, Figure 1 ) is a hormone produced in the pineal gland located in the center of the brain. It is interesting that the secretion of melatonin is not restricted to mammals, but it is also produced in nonmammalian vertebrates, in some invertebrates, and even in many plants, with the same molecular structure [1]. It is one among many endogenous body environment sensors. Interest in this small molecule has increased dramatically in the past several decades. As a result, a number of important biological actions of melatonin have been reported. Several studies have demonstrated that this compound can act as a maternal fetal synchronizer [2] or a chemical mediator of photoperiodic information [3,4]. Melatonin is also a modulator of various endocrinological, neurophysiological, and behavioral functions in vertebrates [5-8]. Especially, melatonin has been shown to regulate retinal vertebrate physiology and to function as an inducer of sleep in humans. In this respect, melatonin acts by a unique mechanism of action by targeting specific receptors in the brain that are responsible for controlling the body's sleep-wake cycle [5]. In addition, this compound can influence the circadian rhythm in reptiles, birds, and mammals including human. Recently, it has been observed that melatonin level is found to decline with age. This observation leads many scientists to believe that the lack of melatonin may play a role in the development of age related disorders. Among the diseases that melatonin is thought to affect are Alzheimer's disease, glucose intolerance, impaired immune function, and cancer [9].

Due to diverse biological properties mentioned above, melatonin has received a great deal of attention in the last decade. Various forms of over-the-counter drugs or food supplements are available with therapeutic indications including synchronization of disturbed circadian rhythms such as jetlag [10], sleep-wake cycle [11], seasonal disorders [12], and winter depression [13].

In clinical setting, it is noted that toxicity of melatonin is remarkably low, and no serious negative side effects of melatonin have been reported, so far [14]. The main disadvantage of melatonin is its poor pharmacokinetic profile. Though the bioavailability of melatonin achieves $30-50 \%$, in vivo, melatonin is quickly metabolized with a half-life of only 30 to 50 minutes [15], which greatly limits its clinical value.

With the aim to improve the pharmacokinetic profile of melatonin, we have designed a series of sustain-acting melatonin prodrugs (Figure 2). This paper reports the results of synthesis and preliminary evaluation of melatonin release from these potential prodrugs. 


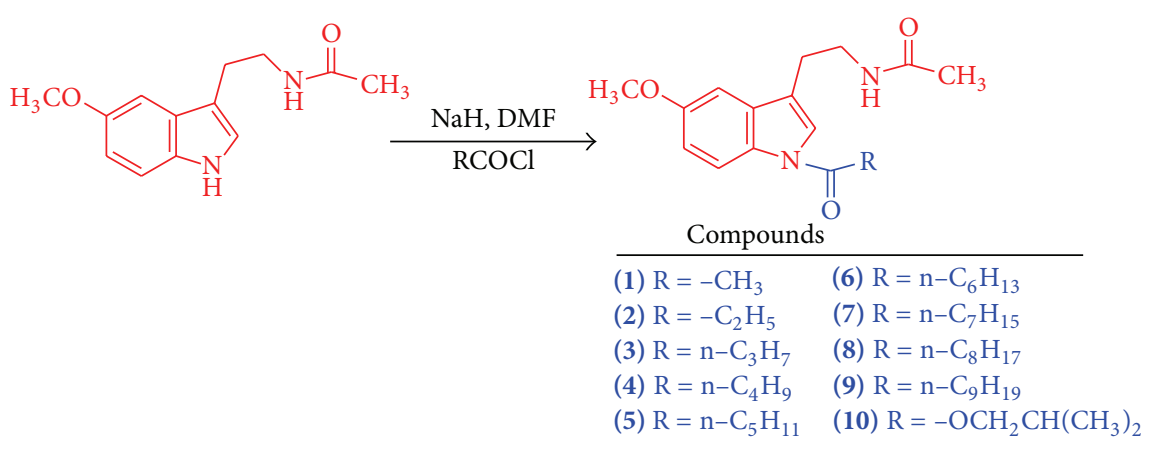

SCHeme 1: Synthesis of $N^{1}$-acylmelatonin derivatives.

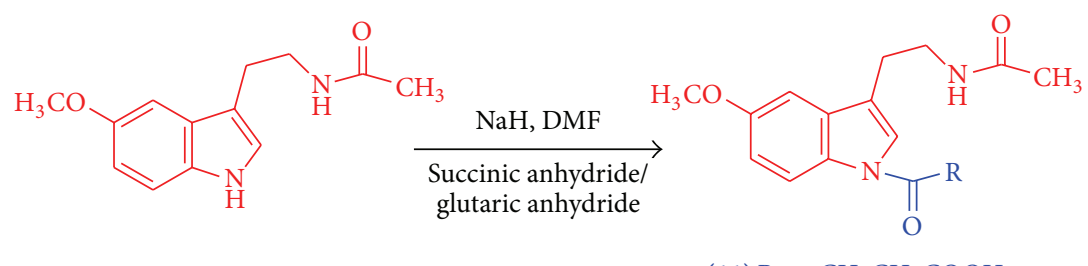

(11) $\mathrm{R}=-\mathrm{CH}_{2} \mathrm{CH}_{2} \mathrm{COOH}$

(12) $\mathrm{R}=-\mathrm{CH}_{2} \mathrm{CH}_{2} \mathrm{CH}_{2} \mathrm{COOH}$

Scheme 2: Synthesis of $N^{1}$-succinoyl and $N^{1}$-glutaroylmelatonin derivatives.<smiles>COc1ccc2[nH]cc(CCNC(C)=O)c2c1</smiles>

Figure 1: Structure of melatonin.

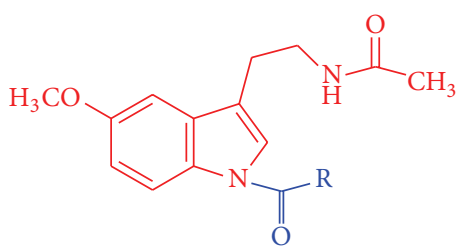

Compounds
(1) $\mathrm{R}=-\mathrm{CH}_{3}$
(7) $\mathrm{R}=\mathrm{n}-\mathrm{C}_{7} \mathrm{H}_{15}$
(2) $\mathrm{R}=-\mathrm{C}_{2} \mathrm{H}_{5}$
(8) $\mathrm{R}=\mathrm{n}-\mathrm{C}_{8} \mathrm{H}_{17}$
(3) $\mathrm{R}=\mathrm{n}-\mathrm{C}_{3} \mathrm{H}_{7}$
(9) $\mathrm{R}=\mathrm{n}-\mathrm{C}_{9} \mathrm{H}_{19}$
(4) $\mathrm{R}=\mathrm{n}-\mathrm{C}_{4} \mathrm{H}_{9}$
(10) $\mathrm{R}=-\mathrm{OCH}_{2} \mathrm{CH}\left(\mathrm{CH}_{3}\right)_{2}$
(5) $\mathrm{R}=\mathrm{n}-\mathrm{C}_{5} \mathrm{H}_{11}$
(11) $\mathrm{R}=-\mathrm{CH}_{2} \mathrm{CH}_{2} \mathrm{COOH}$
(6) $\mathrm{R}=\mathrm{n}-\mathrm{C}_{6} \mathrm{H}_{13}$
(12) $\mathrm{R}=-\mathrm{CH}_{2} \mathrm{CH}_{2} \mathrm{CH}_{2} \mathrm{COOH}$

FIGURE 2: Designed melatonin prodrugs.

\section{Results and Discussion}

Compounds 1-10, the $N^{1}$-acylated melatonin derivatives with acyl chains from 2 to $10 \mathrm{C}(\mathbf{1 - 9})$ or s-butyloxycarbonyl (10), were synthesized from melatonin by reacting with appropriate acyl chlorides in dimethylformamide used as a solvent (Scheme 1). Conventional methods using pyridine or triethylamine as bases were not successful. It was found that sodium hydride gave the best results, affording the target compounds in average to good yields (65-87\%). Among the $N^{1}$-acylated melatonin derivatives (1-9), compound 1 had been synthesized previously [16].

Two additional compounds $\mathbf{1 1}$ and $\mathbf{1 2}$ bearing succinyl and glutaryl moieties were synthesized by a similar protocol, just using succinic anhydride or glutaric anhydride instead of acyl chloride (Scheme 2).

All compounds were unambiguously identified by analysis of spectral data, including IR, MS, ${ }^{1} \mathrm{H}-\mathrm{NMR}$, and ${ }^{13} \mathrm{C}$ NMR. In the IR spectra, all characteristic absorption bands of functional groups clearly appeared, for example, $3325(\mathrm{NH})$, 3010-3220 (CH-aromatic), 2930, $2910\left(\mathrm{CH}_{3}, \mathrm{CH}_{2}\right), \sim 1670-$ $1720(\mathrm{C}=\mathrm{O}), \sim 1610,1580,1475$ (C=C, aromatic), and $\sim 1250$ (C-O). ESI mass spectra showed strong molecular peaks and a few fragments appropriately matching the corresponding structures. In the ${ }^{1} \mathrm{H}-\mathrm{NMR}$ and ${ }^{13} \mathrm{C}-\mathrm{NMR}$, besides the characteristic peaks of the melatonin skeleton, all protons and carbons of the newly added $N^{1}$-acyl moieties were observed.

All the synthesized compounds were preliminarily evaluated for the ability to release melatonin using an $80 \%$ human plasma assay [17]. In these experiments, each compound was incubated with an $80 \%$ human plasma solution ( $\mathrm{pH} 7.4$ ). After each interval of time (1, 2, 3, and 4 hours) an aliquot of $500 \mu \mathrm{L}$ from each compound's solution was withdrawn and processed to give a clear supernatant which was then analyzed by HPLC to determine the melatonin release rate. It was found that compounds $\mathbf{1 - 1 0}$ did not release melatonin efficiently. After 4 hours, the percentages of melatonin release from these compounds achieved only less than 20\% (Table 1). For intended use of these compounds as sleep inducers, these melatonin release percentages were clearly not appropriate. 
TABLE 1: Percentages of melatonin release from compounds 1-12 after incubation with $80 \%$ human plasma for different time intervals.

\begin{tabular}{|c|c|c|c|c|c|}
\hline \multirow{2}{*}{ Cpd } & \multirow{2}{*}{$\mathrm{R}$} & \multicolumn{4}{|c|}{ Percentage (\%) of melatonin released after incubation time of } \\
\hline & & 1 hour & 2 hours & 3 hours & 4 hours \\
\hline 1 & $-\mathrm{CH}_{3}$ & - & $7.5 \pm 1.1$ & $15.4 \pm 1.3$ & $18.9 \pm 1.7$ \\
\hline 2 & $-\mathrm{CH}_{2} \mathrm{CH}_{5}$ & - & $7.5 \pm 1.2$ & $15.8 \pm 1.2$ & $17.8 \pm 1.5$ \\
\hline 3 & $n-\mathrm{C}_{3} \mathrm{H}_{7}$ & - & $7.1 \pm 0.9$ & $14.9 \pm 0.9$ & $19.4 \pm 2.0$ \\
\hline 4 & $n-\mathrm{C}_{4} \mathrm{H}_{9}$ & - & $6.5 \pm 0.7$ & $15.2 \pm 1.3$ & $17.4 \pm 1.9$ \\
\hline 5 & $n-\mathrm{C}_{5} \mathrm{H}_{11}$ & - & $6.7 \pm 0.5$ & $15.0 \pm 1.0$ & $18.5 \pm 2.1$ \\
\hline 6 & $n-\mathrm{C}_{6} \mathrm{H}_{13}$ & - & $6.3 \pm 0.5$ & $14.7 \pm 0.8$ & $17.6 \pm 1.7$ \\
\hline 7 & $n-\mathrm{C}_{7} \mathrm{H}_{15}$ & - & $5.9 \pm 0.5$ & $14.2 \pm 1.4$ & $16.3 \pm 1.0$ \\
\hline 8 & $n-\mathrm{C}_{8} \mathrm{H}_{17}$ & - & $5.6 \pm 0.5$ & $14.4 \pm 1.2$ & $16.0 \pm 1.1$ \\
\hline 9 & $n-\mathrm{C}_{9} \mathrm{H}_{19}$ & - & $5.1 \pm 0.5$ & $13.9 \pm 1.0$ & $15.3 \pm 0.9$ \\
\hline 10 & & $15.7 \pm 1.5$ & $26.9 \pm 2.1$ & $38.2 \pm 2.9$ & $44.7 \pm 3.2$ \\
\hline 11 & & $25.4 \pm 2.2$ & $37.8 \pm 2.9$ & $64.4 \pm 3.2$ & $75.2 \pm 3.8$ \\
\hline 12 & $\mathrm{O}$ & $31.5 \pm 2.4$ & $44.1 \pm 3.7$ & $67.3 \pm 3.5$ & $83.5 \pm 4.2$ \\
\hline
\end{tabular}

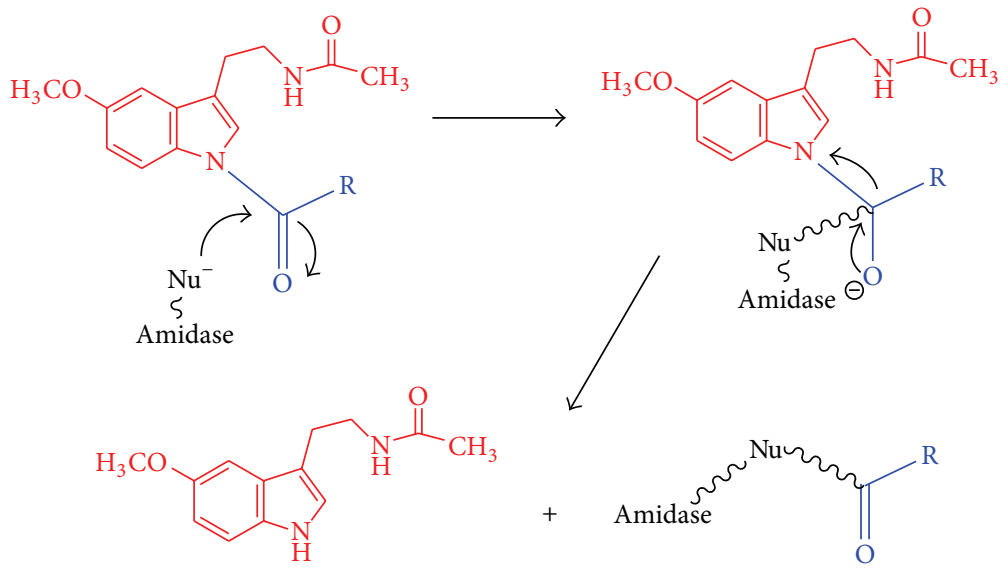

FIGURE 3: Illustration of amidase catalyzed release of melatonin from melatonin derivatives $\left(\mathrm{Nu}^{-}=\mathrm{SH}, \mathrm{NH}_{2}, \mathrm{OH}\right.$, etc.).

Thus, it seemed that a proposed release mechanism for melatonin, catalyzed by amidases (Figure 3), was not efficient enough.

Compound $\mathbf{1 0}$ appeared to have much better melatonin releasing profile, with melatonin release rates achieving $26.9 \pm$ $2.1,44.7 \pm 3.2$, and $44.7 \pm 3.2 \%$ after 2 -, 3-, and 4 -hourincubation. Especially, compounds 11 and 12 showed the most impressive melatonin release rates. After 2-hour incubation time, $37.8 \pm 2.9$ and $44.7 \pm 3.1 \%$ of melatonin were released from compounds $\mathbf{1 1}$ and 12, respectively. These data after 3 -hour incubation time were $67.3 \pm 3.5$, and $67.3 \pm 3.5 \%$; and after 3 -hour incubation were $75.2 \pm 3.8$ and $83.5 \pm 4.2$, respectively (Table 1). The more efficient release of melatonin from compounds 11 and 12 could be speculated, in part, by the self-immolation assisted hydrolysis (Figure 4). This property has been commonly observed with succinyl and glutaroyl prodrugs and other similar ones [18-20]. From these results, it is expected that compounds $\mathbf{1 1}$ and $\mathbf{1 2}$ could serve as promising sustained release prodrugs of melatonin for using as natural-sleep inducers.

\section{Conclusion}

We have designed and synthesized twelve melatonin amide prodrugs aiming at prolonging the action of melatonin 


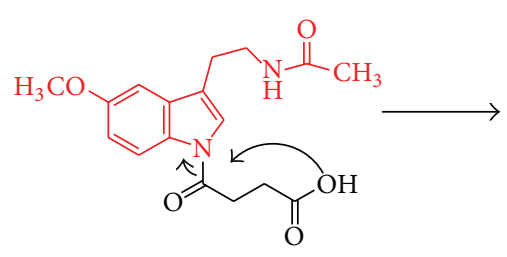<smiles>COc1ccc2[nH]cc(CCNC(C)=O)c2c1</smiles>
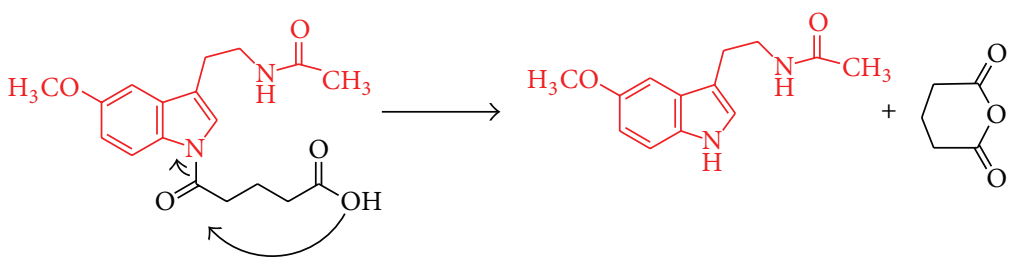

FIGURE 4: Illustration of self-immolation release of melatonin from compounds $\mathbf{1 1}$ and $\mathbf{1 2 .}$

in vivo by improving its half-life. The $N^{1}$-succinyl and $N^{1}$ glutaroylmelatonin derivatives (compounds 11 and 12) could be promising as sustained release melatonin prodrugs.

\section{Experimental Protocols}

All products were homogenous, as examined by thin-layer chromatography (TLC), performed on Whatman $250 \mu \mathrm{m}$ Silica Gel GF Uniplates and visualized under UV light at 254 and $365 \mathrm{~nm}$. Melting points were determined with an Electrothermal Melting Point apparatus and are uncorrected. Chromatographic purification was done by the open flash silica gel column chromatography using Merck silica gel 60 (240 to 400 mesh). Nuclear magnetic resonance spectra $\left({ }^{1} \mathrm{H}-\mathrm{NMR}\right)$ were recorded using tetramethylsilane as an internal standard on a Bruker $500 \mathrm{MHz}$ spectrometer with DMSO- $d_{6}$ as solvent unless otherwise indicated. Chemical shifts are reported in parts per million (ppm) downfield from tetramethylsilane as internal standard. Electron ionization (EI), electrospray ionization (ESI), and high-resolution mass spectra were obtained using PE Biosystems API 2000 and Mariner mass spectrometers, respectively. Reagents and solvents were purchased from Aldrich or Fluka Chemical Corp. (Milwaukee, WI, USA) or Merck unless noted otherwise. Solvents were distilled and dried before use. Elemental analysis results were found within $\pm 0.3 \%$.

General Procedures for Synthesis of Compounds 1-10. In a $50 \mathrm{~mL}$ round bottom flask, sodium hydride was placed (120 mg, $3 \mathrm{mmoL}, 60 \%$ in oil). Approximately $3 \mathrm{~mL}$ of $\mathrm{n}-$ hexane (anhydrous) was added. After gentle shaking, the nhexane layer was discarded. A solution of melatonin ( $0.46 \mathrm{~g}$, $2 \mathrm{mmoL}$ ) dissolved in $5 \mathrm{~mL}$ of dimethylformamide (DMF) was added in portion to the reaction flask. The resulting mixture was stirred at room temperature for 30 minutes then cooled to $0-5^{\circ} \mathrm{C}$ in an ice bath. Then, acyl chloride ( $3 \mathrm{mmoL}$ ) was dropwise added over 10-15 minutes into the reaction mixture to maintain the temperature in the reaction flask below $5^{\circ} \mathrm{C}$. After the addition was complete, the temperature of the reaction mixture was raised to about $80-$ $100^{\circ} \mathrm{C}$, and stirring was continued for about 24 hours. After that, the reaction mixture was cooled and slowly transferred into a cold solution of sodium bicarbonate $(10 \%, 100 \mathrm{~mL})$. Precipitates were collected and recrystallized from n-hexaneacetone to furnish the target compounds 1-10.

N-(2-(1-Acetyl-5-methoxy-1H-indol-3-yl)ethyl)acetamide (1). White solid; Yield: $87 \%$; Mp: $144-147^{\circ} \mathrm{C}$; IR $\left(\mathrm{KBr}, \mathrm{cm}^{-1}\right)$ : $3325(\mathrm{NH}), 3020$ (CH-aromatic), 2930, $2910\left(\mathrm{CH}_{3}, \mathrm{CH}_{2}\right)$, 1715, $1680(\mathrm{C}=\mathrm{O}), 1618,1583,1481(\mathrm{C}=\mathrm{C}$, aromatic $), 1250$ $(\mathrm{C}-\mathrm{O})$. ESI-MS $(\mathrm{m} / \mathrm{z}): 275[\mathrm{M}+\mathrm{H}]^{+}, 232\left[\mathrm{M}-\mathrm{CH}_{3} \mathrm{CO}\right]^{+}$, $190\left[\mathrm{M}-2 \mathrm{CH}_{3} \mathrm{CO}\right]^{+}, 160\left[\mathrm{M}-2 \mathrm{CH}_{3} \mathrm{CO}-\mathrm{OCH}_{3}\right]^{+} .{ }^{1} \mathrm{H}-\mathrm{NMR}$ $\left(500 \mathrm{MHz}, \mathrm{DMSO}-d_{6}, \mathrm{ppm}\right): \delta 7.36(\mathrm{~s}, 1 \mathrm{H}), 7.09(d, 1 \mathrm{H}$, $J=8.20 \mathrm{~Hz}), 6.67(1 \mathrm{H}, d, J=8.20 \mathrm{~Hz}), 6.63(\mathrm{~s}, 1 \mathrm{H}), 3.71(\mathrm{~s}$, $3 \mathrm{H}), 3.46-2.66$ (4H, m), 2.10 (s, 3H), 2.07 (s, 3H). ${ }^{13} \mathrm{C}-\mathrm{NMR}$ (125 MHz, DMSO- $d_{6}$, ppm): $\delta$ 169.89, 167.07, 157.65, 131.24, 128.52, 125.72, 113.32, 110.43, 109.23, 104.32, 57.80, 24.31, 23.25 . Anal. $\mathrm{C}_{15} \mathrm{H}_{18} \mathrm{~N}_{2} \mathrm{O}_{3}$ : C, H, N.

Synthesis of N-(2-(5-Methoxy-1-propionyl-1H-indol-3-yl)ethyl) hyl)acetamide (2). White solid; Yield: 80\%; Mp: 152$154^{\circ} \mathrm{C}$; IR $\left(\mathrm{KBr}, \mathrm{cm}^{-1}\right): 3330(\mathrm{NH}), 3015$ (CH-aromatic), 2925, $2910\left(\mathrm{CH}_{3}, \mathrm{CH}_{2}\right), 1720,1680(\mathrm{C}=\mathrm{O}), 1620,1580$, 1475 (C=C, aromatic), 1251 (C-O). ESI-MS (m/z): 289 $[\mathrm{M}+\mathrm{H}]^{+}, 246\left[\mathrm{M}-\mathrm{CH}_{3} \mathrm{CO}\right]^{+}, 190\left[\mathrm{M}-\mathrm{CH}_{3} \mathrm{CO}-\mathrm{C}_{2} \mathrm{H}_{5} \mathrm{CO}\right]^{+}$, $160\left[\mathrm{M}-\mathrm{CH}_{3} \mathrm{CO}-\mathrm{C}_{2} \mathrm{H}_{5} \mathrm{CO}-\mathrm{OCH}_{3}\right]^{+} .{ }^{1} \mathrm{H}-\mathrm{NMR} \quad(500 \mathrm{MHz}$, DMSO- $\left.d_{6}, \mathrm{ppm}\right): \delta 7.46(\mathrm{~s}, 1 \mathrm{H}), 7.22(d, 1 \mathrm{H}, J=8.25 \mathrm{~Hz})$, $6.71(1 \mathrm{H}, d, J=8.25 \mathrm{~Hz}), 6.69(\mathrm{~s}, 1 \mathrm{H}), 3.82(\mathrm{~s}, 3 \mathrm{H}), 3.47-2.69$ $(4 \mathrm{H}, \mathrm{m}), 2.47(2 \mathrm{H}, \mathrm{m}), 2.15(\mathrm{~s}, 3 \mathrm{H}), 2.11(\mathrm{~m}, 3 \mathrm{H}) .{ }^{13} \mathrm{C}-\mathrm{NMR}$ (125 MHz, DMSO- $\left.d_{6}, \mathrm{ppm}\right): \delta 170.45,169.45,156.34,130.34$, $129.42,126,46,112.32,111.78,110.32,103.38,56.43,28.67,24.31$, 15.47. Anal. $\mathrm{C}_{16} \mathrm{H}_{20} \mathrm{~N}_{2} \mathrm{O}_{3}$ : C, H, N.

Synthesis of N-(2-(1-Butyryl-5-methoxy-1H-indol-3-yl)ethyl) acetamide (3). White solid; Yield: $84 \%$; Mp: $138-141^{\circ} \mathrm{C}$; IR $\left(\mathrm{KBr}, \mathrm{cm}^{-1}\right)$ : $3330(\mathrm{NH}), 3015$ (CH-aromatic), 2925, 2910 $\left(\mathrm{CH}_{3}, \mathrm{CH}_{2}\right), 1710,1670(\mathrm{C}=\mathrm{O}), 1620,1580,1475(\mathrm{C}=\mathrm{C}$, aromatic), 1251 (C-O). ESI-MS (m/z): $303[\mathrm{M}+\mathrm{H}]^{+}$, $260\left[_{\left.\mathrm{M}-\mathrm{CH}_{3} \mathrm{CO}\right]^{+},} 190\left[\mathrm{M}-\mathrm{CH}_{3} \mathrm{CO}-\mathrm{C}_{3} \mathrm{H}_{7} \mathrm{CO}\right]^{+}, \quad 160\right.$ $\left[\mathrm{M}-\mathrm{CH}_{3} \mathrm{CO}-\mathrm{C}_{3} \mathrm{H}_{7} \mathrm{CO}-\mathrm{OCH}_{3}\right]^{+}$. ${ }^{1} \mathrm{H}-\mathrm{NMR} \quad(500 \mathrm{MHz}$, DMSO- $\left.d_{6}, \mathrm{ppm}\right): \delta 7.39(\mathrm{~s}, 1 \mathrm{H}), 7.17(d, 1 \mathrm{H}, J=7.89 \mathrm{~Hz})$, $6.73(1 \mathrm{H}, d, J=7.89 \mathrm{~Hz}), 6.65(\mathrm{~s}, 1 \mathrm{H}), 3.77(\mathrm{~s}, 3 \mathrm{H}), 3.41-2.60$ $(4 \mathrm{H}, \mathrm{m}), 2.45(2 \mathrm{H}, \mathrm{m}), 2.06(\mathrm{~m}, 3 \mathrm{H}), 1.63(\mathrm{~m}, 2 \mathrm{H}), 0.99$ $(\mathrm{m}, 3 \mathrm{H})$. 
Synthesis of N-(2-(5-Methoxy-1-pentanoyl-1Hindol-3-yl)ethyl) acetamide (4). White solids; Yield: $75 \%$; Mp: $157-160^{\circ} \mathrm{C}$; IR $\left(\mathrm{KBr}, \mathrm{cm}^{-1}\right): 3215(\mathrm{NH}), 3020(\mathrm{CH}$-aromatic), 2930, $2915\left(\mathrm{CH}_{3}, \mathrm{CH}_{2}\right), 1700,1675(\mathrm{C}=\mathrm{O}), 1610,1572,1479$ $\left(\mathrm{C}=\mathrm{C}\right.$, aromatic), 1251, 1210. ESI-MS $(\mathrm{m} / \mathrm{z}): 321[\mathrm{M}+\mathrm{H}]^{+}$, $274\left[\mathrm{M}-\mathrm{CH}_{3} \mathrm{CO}\right]^{+}, \quad 190 \quad\left[\mathrm{M}-\mathrm{CH}_{3} \mathrm{CO}-\mathrm{C}_{4} \mathrm{H}_{9} \mathrm{CO}\right]^{+}, \quad 160$ $\left[\mathrm{M}-\mathrm{CH}_{3} \mathrm{CO}-\mathrm{C}_{4} \mathrm{H}_{9} \mathrm{CO}-\mathrm{OCH}_{3}\right]^{+}$. ${ }^{1} \mathrm{H}-\mathrm{NMR} \quad(500 \mathrm{MHz}$, DMSO- $\left.d_{6}, \mathrm{ppm}\right): \delta 7.41(\mathrm{~s}, 1 \mathrm{H}), 7.13(d, 1 \mathrm{H}, J=7.90 \mathrm{~Hz}), 6.71$ $(1 \mathrm{H}, d, J=7.90 \mathrm{~Hz}), 6.69(\mathrm{~s}, 1 \mathrm{H}), 3.79(\mathrm{~s}, 3 \mathrm{H}), 3.45-2.63(4 \mathrm{H}$, $\mathrm{m}), 2.45(2 \mathrm{H}, \mathrm{m}), 2.10(\mathrm{~m}, 3 \mathrm{H}), 1.64-1.37(\mathrm{~m}, 4 \mathrm{H}), 0.97(\mathrm{~m}$, $3 \mathrm{H})$.

Synthesis of N-(2-(1-Hexanoyl-5-methoxy-1H-indol-3-yl)ethyl) acetamide (5). White solid; Yield: $79 \%$; Mp: $147-149^{\circ} \mathrm{C}$; IR $\left(\mathrm{KBr}, \mathrm{cm}^{-1}\right): 3227(\mathrm{NH}), 3030$ (CH-aromatic), 2950, 2915, $2910\left(\mathrm{CH}_{3}, \mathrm{CH}_{2}\right), 1695,1675(\mathrm{C}=\mathrm{O}), 1630,1590,1470$ $\left(\mathrm{C}=\mathrm{C}\right.$, aromatic), $1250(\mathrm{C}-\mathrm{O})$. ESI-MS (m/z): $335[\mathrm{M}+\mathrm{H}]^{+}$, $288\left[\mathrm{M}-\mathrm{CH}_{3} \mathrm{CO}\right]^{+}, 190\left[\mathrm{M}-\mathrm{CH}_{3} \mathrm{CO}-\mathrm{C}_{5} \mathrm{H}_{11} \mathrm{CO}\right]^{+}, \quad 160$ $\left[\mathrm{M}-\mathrm{CH}_{3} \mathrm{CO}-\mathrm{C}_{5} \mathrm{H}_{11} \mathrm{CO}-\mathrm{OCH}_{3}\right]^{+}$. ${ }^{1} \mathrm{H}-\mathrm{NMR} \quad(500 \mathrm{MHz}$, DMSO- $\left.d_{6}, \mathrm{ppm}\right): \delta 7.39(\mathrm{~s}, 1 \mathrm{H}), 7.11(d, 1 \mathrm{H}, J=7.80 \mathrm{~Hz}), 6.75$ $(1 \mathrm{H}, d, J=7.80 \mathrm{~Hz}), 6.73(\mathrm{~s}, 1 \mathrm{H}), 3.81(\mathrm{~s}, 3 \mathrm{H}), 3.47-2.66(4 \mathrm{H}$, $\mathrm{m}), 2.47(2 \mathrm{H}, \mathrm{m}), 2.11(\mathrm{~m}, 3 \mathrm{H}), 1.65-1.33(\mathrm{~m}, 6 \mathrm{H}), 0.96(\mathrm{~m}$, $3 \mathrm{H})$.

Synthesis of N-(2-(1-Heptanoyl-5-methoxy-1H-indol-3-yl)ethyl) acetamide (6). White solid; Yield: $68 \%$; Mp: $146-148^{\circ} \mathrm{C}$; IR $\left(\mathrm{KBr}, \mathrm{cm}^{-1}\right): 3310(\mathrm{NH}), 3020$ (CH-aromatic), 2905, $2900\left(\mathrm{CH}_{3}, \mathrm{CH}_{2}\right), 1710,1670(\mathrm{C}=\mathrm{O}), 1600,1574,1482(\mathrm{C}=\mathrm{C}$, aromatic), $1253(\mathrm{C}-\mathrm{O})$. ESI-MS $(\mathrm{m} / \mathrm{z}): 349[\mathrm{M}+\mathrm{H}]^{+}$, $302\left[\mathrm{M}-\mathrm{CH}_{3} \mathrm{CO}\right]^{+}, 190\left[\mathrm{M}-\mathrm{CH}_{3} \mathrm{CO}-\mathrm{C}_{6} \mathrm{H}_{13} \mathrm{CO}\right]^{+}, 160$ $\left[\mathrm{M}-\mathrm{CH}_{3} \mathrm{CO}-\mathrm{C}_{6} \mathrm{H}_{13} \mathrm{CO}-\mathrm{OCH}_{3}\right]^{+}$. ${ }^{1} \mathrm{H}-\mathrm{NMR} \quad(500 \mathrm{MHz}$, DMSO- $\left.d_{6}, \mathrm{ppm}\right): \delta 7.36(\mathrm{~s}, 1 \mathrm{H}), 7.09(d, 1 \mathrm{H}, J=8.12 \mathrm{~Hz})$, $6.79(1 \mathrm{H}, d, J=8.12 \mathrm{~Hz}), 6.71(\mathrm{~s}, 1 \mathrm{H}), 3.74(\mathrm{~s}, 3 \mathrm{H}), 3.41-2,62$ $(4 \mathrm{H}, \mathrm{m}), 2.46(2 \mathrm{H}, \mathrm{m}), 2.04(\mathrm{~m}, 3 \mathrm{H}), 1.62-1.34(\mathrm{~m}, 8 \mathrm{H}), 0.98$ $(\mathrm{m}, 3 \mathrm{H})$.

Synthesis of N-(2-(5-Methoxy-1-octanoyl-1H-indol-3-yl)ethyl) acetamide (7). White solid; Yield: $71 \%$; Mp: $140-142^{\circ} \mathrm{C}$; IR $\left(\mathrm{KBr}, \mathrm{cm}^{-1}\right): 3150(\mathrm{NH}), 3003$ (CH-aromatic), 2950, 2925 $\left(\mathrm{CH}_{3}, \mathrm{CH}_{2}\right), 1690,1665(\mathrm{C}=\mathrm{O}), 1620,1570,1480(\mathrm{C}=\mathrm{C}$, aromatic), 1250, $1210(\mathrm{C}-\mathrm{O})$. ESI-MS $(\mathrm{m} / \mathrm{z}): 363[\mathrm{M}+\mathrm{H}]^{+}$, $316\left[\mathrm{M}-\mathrm{CH}_{3} \mathrm{CO}\right]^{+}, \quad 190 \quad\left[\mathrm{M}-\mathrm{CH}_{3} \mathrm{CO}-\mathrm{C}_{7} \mathrm{H}_{15} \mathrm{CO}\right]^{+}, \quad 160$ $\left[\mathrm{M}-\mathrm{CH}_{3} \mathrm{CO}-\mathrm{C}_{7} \mathrm{H}_{15} \mathrm{CO}-\mathrm{OCH}_{3}\right]^{+}$. ${ }^{1} \mathrm{H}-\mathrm{NMR} \quad(500 \mathrm{MHz}$, DMSO- $\left.d_{6}, \mathrm{ppm}\right): \delta 7.41(\mathrm{~s}, 1 \mathrm{H}), 7.11(d, 1 \mathrm{H}, J=8.11 \mathrm{~Hz}), 6.83$ $(1 \mathrm{H}, d, J=8.11 \mathrm{~Hz}), 6.74(\mathrm{~s}, 1 \mathrm{H}), 3.77(\mathrm{~s}, 3 \mathrm{H}), 3.44-2.65(4 \mathrm{H}$, m), $2.47(2 \mathrm{H}, \mathrm{m}), 2.09(\mathrm{~m}, 3 \mathrm{H}), 1.67-1.33(\mathrm{~m}, 10 \mathrm{H}), 0.99(\mathrm{~m}$, $3 \mathrm{H})$.

Synthesis of N-(2-(5-Methoxy-1-nonanoyl-1H-indol-3-yl)ethyl) acetamide (8). White solid; Yield: $65 \%$; $\mathrm{Mp}: 139-143^{\circ} \mathrm{C}$; IR $\left(\mathrm{KBr}, \quad \mathrm{cm}^{-1}\right): 3225(\mathrm{NH}), 3023 \quad(\mathrm{CH}$-aromatic), 2945, 2915, $2910\left(\mathrm{CH}_{3}, \mathrm{CH}_{2}\right), 1695,1672(\mathrm{C}=\mathrm{O}), 1630$, 1590, 1470, 1250 (C-O). ESI-MS (m/z): $377[\mathrm{M}+\mathrm{H}]^{+}$, $330\left[\mathrm{M}-\mathrm{CH}_{3} \mathrm{CO}\right]^{+}, \quad 190 \quad\left[\mathrm{M}-\mathrm{CH}_{3} \mathrm{CO}-\mathrm{C}_{8} \mathrm{H}_{17} \mathrm{CO}\right]^{+}, \quad 160$ $\left[\mathrm{M}-\mathrm{CH}_{3} \mathrm{CO}-\mathrm{C}_{8} \mathrm{H}_{17} \mathrm{CO}-\mathrm{OCH}_{3}\right]^{+}$. ${ }^{1} \mathrm{H}-\mathrm{NMR} \quad(500 \mathrm{MHz}$, DMSO- $\left.d_{6}, \mathrm{ppm}\right): \delta 7.47(\mathrm{~s}, 1 \mathrm{H}), 7.24(d, 1 \mathrm{H}, J=8.32 \mathrm{~Hz})$, $6.89(1 \mathrm{H}, d, J=8.32 \mathrm{~Hz}), 6.79(\mathrm{~s}, 1 \mathrm{H}), 3.91(\mathrm{~s}, 3 \mathrm{H}), 3.51-2.69$
$(4 \mathrm{H}, \mathrm{m}), 2.52(2 \mathrm{H}, \mathrm{m}), 2.12(\mathrm{~m}, 3 \mathrm{H}), 1.69-1.30(\mathrm{~m}, 12 \mathrm{H}), 1.00$ $(\mathrm{m}, 3 \mathrm{H})$.

Synthesis of N-(2-(1-Decanoyl-5-methoxy-1H-indol-3-yl)ethyl) acetamide (9). White solid; Yield: $63 \%$; $\mathrm{Mp}: 155-158^{\circ} \mathrm{C}$; IR $\left(\mathrm{KBr}, \mathrm{cm}^{-1}\right)$ : $3240(\mathrm{NH}), 3020$ (CH-aromatic), 2945, 2935, $2915\left(\mathrm{CH}_{3}, \mathrm{CH}_{2}\right), 1710,1670(\mathrm{C}=\mathrm{O}), 1620,1580$, $1485\left(\mathrm{C}=\mathrm{C}\right.$, aromatic), 1250. ESI-MS $(\mathrm{m} / \mathrm{z}): 391[\mathrm{M}+\mathrm{H}]^{+}$, $344\left[\mathrm{M}-\mathrm{CH}_{3} \mathrm{CO}\right]^{+}, \quad 190 \quad\left[\mathrm{M}-\mathrm{CH}_{3} \mathrm{CO}-\mathrm{C}_{9} \mathrm{H}_{19} \mathrm{CO}\right]^{+}, 160$ $\left[\mathrm{M}-\mathrm{CH}_{3} \mathrm{CO}-\mathrm{C}_{9} \mathrm{H}_{19} \mathrm{CO}-\mathrm{OCH}_{3}\right]^{+} .{ }^{1} \mathrm{H}-\mathrm{NMR} \quad(500 \mathrm{MHz}$, DMSO- $\left.d_{6}, \mathrm{ppm}\right): \delta 7.42(\mathrm{~s}, 1 \mathrm{H}), 7.21(d, 1 \mathrm{H}, J=8.29 \mathrm{~Hz})$, $6.92(1 \mathrm{H}, d, J=8.29 \mathrm{~Hz}), 6.81(\mathrm{~s}, 1 \mathrm{H}), 3.99(\mathrm{~s}, 3 \mathrm{H}), 3.52-2.71$ $(4 \mathrm{H}, \mathrm{m}), 2.57(2 \mathrm{H}, \mathrm{m}), 2.10(\mathrm{~m}, 3 \mathrm{H}), 1.71-1.32(\mathrm{~m}, 14 \mathrm{H}), 1.02$ $(\mathrm{m}, 3 \mathrm{H})$.

Synthesis of Isobutyl 3-(2-Acetamidoethyl)-5-methoxy-1H-indole-1-carboxylate (10). White solid; Yield: 75\%; Mp: 167$169^{\circ} \mathrm{C}$; IR $\left(\mathrm{KBr}, \mathrm{cm}^{-1}\right)$ : $3250(\mathrm{NH}), 3050$ (CH-aromatic), 2930, $2910\left(\mathrm{CH}_{3}, \mathrm{CH}_{2}\right), 16950,1660(\mathrm{C}=\mathrm{O}), 1610,1545,1480$ $\left(\mathrm{C}=\mathrm{C}\right.$, aromatic), $1220(\mathrm{C}-\mathrm{O})$. ESI-MS $(\mathrm{m} / \mathrm{z}): 333[\mathrm{M}+\mathrm{H}]^{+}$, $290\left[\mathrm{M}-\mathrm{CH}_{3} \mathrm{CO}\right]^{+}, 190\left[\mathrm{M}-\mathrm{CH}_{3} \mathrm{CO}-\mathrm{C}_{4} \mathrm{H}_{9} \mathrm{CO}_{2}\right]^{+}, \quad 160$ $\left[\mathrm{M}-\mathrm{CH}_{3} \mathrm{CO}-\mathrm{C}_{4} \mathrm{H}_{9} \mathrm{CO}_{2}-\mathrm{OCH}_{3}\right]^{+}$. ${ }^{1} \mathrm{H}-\mathrm{NMR} \quad(500 \mathrm{MHz}$, DMSO- $\left.d_{6}, \mathrm{ppm}\right): \delta 7.35(\mathrm{~s}, 1 \mathrm{H}), 7.15(d, 1 \mathrm{H}, J=8.50 \mathrm{~Hz}), 6.95$ $(1 \mathrm{H}, d, J=8.50 \mathrm{~Hz}), 6.82(\mathrm{~s}, 1 \mathrm{H}), 4.09-4.01(2 \mathrm{H}, \mathrm{m}), 3.81$ $(\mathrm{s}, 3 \mathrm{H}), 3.47-3.41(\mathrm{~m}, 2 \mathrm{H}), 2.65-2.45(\mathrm{~m}, 4 \mathrm{H}), 2.10(\mathrm{~m}, 3 \mathrm{H})$, 1.07-1.01 (m, 6H). Anal. $\mathrm{C}_{18} \mathrm{H}_{24} \mathrm{~N}_{2} \mathrm{O}_{4}: \mathrm{C}, \mathrm{H}, \mathrm{N}$.

Synthesis of 4-(3-(2-Acetamidoethyl)-5-methoxy-1H-indol-1yl)-4-oxobutanoic Acid (11). In a $50 \mathrm{~mL}$ round bottom flask sodium hydride was placed $(120 \mathrm{mg}, 3 \mathrm{mmoL}, 60 \%$ in oil). Approximately $3 \mathrm{~mL}$ of $\mathrm{n}$-hexane (anhydrous) was added. After gentle shaking, the $\mathrm{n}$-hexane layer was discarded. A solution of melatonin $(0.46 \mathrm{~g}, 2 \mathrm{mmoL})$ dissolved in $5 \mathrm{~mL}$ of dimethylformamide (DMF) was added in portion to the reaction flask. The resulting mixture was stirred at room temperature for 30 minutes then cooled to $0-5^{\circ} \mathrm{C}$ in an ice-bath. Then, succinic anhydride $(2.2 \mathrm{mmoL})$ was added in portions into the reaction mixture. After the addition was complete, the temperature of the reaction mixture was raised to about $80-100^{\circ} \mathrm{C}$, and stirring was continued for about 24 hours. After that, the reaction mixture was cooled and slowly transferred into $50 \mathrm{~mL}$ of crushed-ice water. $\mathrm{pH}$ was adjusted to 2 by a solution of $5 \% \mathrm{HCl}$. Precipitates were collected and recrystallized from ethanol to furnish the target compound.

White solid; Yield: $66 \%$; Mp: $166-170^{\circ} \mathrm{C}$; IR $\left(\mathrm{KBr}, \mathrm{cm}^{-1}\right)$ : 3450, $3300(\mathrm{OH}), 3220(\mathrm{NH}), 3033(\mathrm{CH}$-aromatic), 2940, 2930, $2910\left(\mathrm{CH}_{3}, \mathrm{CH}_{2}\right), 1725,1680,1675(\mathrm{C}=\mathrm{O}), 1615,1580$, $1485(\mathrm{C}=\mathrm{C}$, aromatic), $1250(\mathrm{C}-\mathrm{O})$. ESI-MS $(\mathrm{m} / \mathrm{z}): 333$ $[\mathrm{M}+\mathrm{H}], 290\left[\mathrm{M}-\mathrm{CH}_{3} \mathrm{CO}\right], 246\left[\mathrm{M}-\mathrm{CH}_{3} \mathrm{CO}-\mathrm{COOH}\right], 190$ $\left(\mathrm{M}-\mathrm{CH}_{3} \mathrm{CO}-\mathrm{COCH}_{2} \mathrm{CH}_{2} \mathrm{COOH}\right), 160\left[\mathrm{M}-\mathrm{CH}_{3} \mathrm{CO}-\mathrm{COC}\right.$ $\left.\mathrm{H}_{2} \mathrm{CH}_{2} \mathrm{COOH}-\mathrm{OCH}_{3}\right] .{ }^{1} \mathrm{H}-\mathrm{NMR}\left(500 \mathrm{MHz}, \mathrm{DMSO}-d_{6}\right.$, ppm): $\delta 7.39(\mathrm{~s}, 1 \mathrm{H}), 7.11(d, 1 \mathrm{H}, J=8.21 \mathrm{~Hz}), 6.71(1 \mathrm{H}$, $d, J=8.21 \mathrm{~Hz}), 6.67(\mathrm{~s}, 1 \mathrm{H}), 3.88(\mathrm{~s}, 3 \mathrm{H}), 3.47-2.63(4 \mathrm{H}, \mathrm{m})$, 2.67-2.57 (m, 4H), $2.11(\mathrm{~s}, 3 \mathrm{H}) .{ }^{13} \mathrm{C}-\mathrm{NMR}(125 \mathrm{MHz}$, DMSO$\left.d_{6}, \mathrm{ppm}\right): \delta 175.90,170.31,169.21,155.35,130.24,129.84,126.42$, $112.42,109.32,108.12,103.45,56.89,42.28,34.89,32.78,28.77$, 24.23. Anal. $\mathrm{C}_{17} \mathrm{H}_{20} \mathrm{~N}_{2} \mathrm{O}_{5}$ : C, $\mathrm{H}, \mathrm{N}$. 
Synthesis of 5-(3-(2-Acetamidoethyl)-5-methoxy-1H-indol-1yl)-5-oxopentanoic Acid (12). This compound was synthesized by a similar procedure used for the synthesis of compound $\mathbf{1 1}$ but using glutaric anhydride instead of succinic anhydride.

White solid; Yield: $64 \%$; Mp: $163-164^{\circ} \mathrm{C}$; IR $(\mathrm{KBr}$, $\left.\mathrm{cm}^{-1}\right): 3440,3320,2935,2925,2915\left(\mathrm{CH}_{3}, \mathrm{CH}_{2}\right), 1720$, $1685(\mathrm{C}=\mathrm{O}), 1620,1615,1587,1482(\mathrm{C}=\mathrm{C}$, aromatic), 1255 $(\mathrm{C}-\mathrm{O})$. ESI-MS $(\mathrm{m} / \mathrm{z}): 34[\mathrm{M}+\mathrm{H}]^{+}, 304\left[\mathrm{M}-\mathrm{CH}_{3} \mathrm{CO}\right]^{+}$, $190\left[\mathrm{M}-\mathrm{CH}_{3} \mathrm{CO}-\mathrm{COCH}_{2} \mathrm{CH}_{2} \mathrm{CH}_{2} \mathrm{COOH}\right]^{+}, 160\left[\mathrm{M}-\mathrm{CH}_{3}\right.$ $\left.\mathrm{CO}-\mathrm{COCH}_{2} \mathrm{CH}_{2} \mathrm{CH}_{2} \mathrm{COOH}-\mathrm{OCH}_{3}\right]^{+}{ }^{1} \mathrm{H}-\mathrm{NMR}(500 \mathrm{MHz}$, DMSO- $\left.d_{6}, \mathrm{ppm}\right): \delta 7.43(\mathrm{~s}, 1 \mathrm{H}), 7.17(d, 1 \mathrm{H}, J=8.23 \mathrm{~Hz})$, $6.75(1 \mathrm{H}, d, J=8.23 \mathrm{~Hz}), 6.69(\mathrm{~s}, 1 \mathrm{H}), 3.89(\mathrm{~s}, 3 \mathrm{H}), 3.49-$ $2.66(4 \mathrm{H}, \mathrm{m}), 2.49-2.21(\mathrm{~m}, 6 \mathrm{H}), 2.10(\mathrm{~s}, 3 \mathrm{H}) .{ }^{13} \mathrm{C}-\mathrm{NMR}$ (125 MHz, DMSO- $\left.d_{6}, \mathrm{ppm}\right): \delta 176.80,169.67,168.43,156.85$, $129.90,128.44,127.42,111.32,110.92,107.87,104.65,57.65,42.25$, 36.55, 32.66, 29.66, 24.33, 21.78. Anal. $\mathrm{C}_{18} \mathrm{H}_{22} \mathrm{~N}_{2} \mathrm{O}_{5}$ : C, H, N.

Determination of the Hydrolysis Percentages of Compounds. The hydrolysis percentage of each compound in an $80 \%$ human plasma ( $\mathrm{pH}$ 7.4) was determined using previously reported method [16]. Briefly, a solution of $20 \mathrm{mg}$ of melatonin or its derivatives was prepared in acetonitrile $(5 \mathrm{~mL})$ and was added to $95 \mathrm{~mL}$ of $80 \%$ human plasma ( $\mathrm{pH} 7.4$, prepared by mixing plasma with phosphate buffer $\mathrm{pH} 7.4$ in a ratio of 4 to 1). An aliquot of $10 \mathrm{~mL}$ of this solution was withdrawn and kept in test tubes maintained at $37 \pm$ $0.5^{\circ} \mathrm{C}$. After each interval of time $(1,2,3$, and 4 hours) an aliquot of $500 \mu \mathrm{L}$ was withdrawn from each tube and transferred to a microcentrifuge tube (Eppendorf's tube). The tubes were placed in freezing mixture in order to stop any further hydrolysis, followed by vortexing thoroughly for $5 \mathrm{~min}$. After vortexing, the tubes were centrifuged at high speed $(10000 \mathrm{rpm})$ for $5 \mathrm{~min}$. A clear supernatant $(20 \mathrm{~mL})$ obtained from each tube was used for HPLC analysis with ODS C18 column, mobile phase including acetonitrile and water (45:65), and UV detector set at $257 \mathrm{~nm}$ wavelength to detect peaks of melatonin and its derivatives. Compounds concentration and percentage in a mixture were quantified by peak area calculation and analysis.

\section{Conflict of Interests}

The authors declare no conflict of interests.

\section{References}

[1] S. R. Pandi-Perumal, V. Srinivasan, G. J. M. Maestroni, D. P. Cardinali, B. Poeggeler, and R. Hardeland, "Melatonin: nature's most versatile biological signal?” FEBS Journal, vol. 273, no. 13, pp. 2813-2838, 2006.

[2] R. Y. Moore, "Development of the suprachiasmatic nucleus," in Suprachiasmatic Nucleus: The Minds'Clock, D. C. Kelvin, R. Y. Moore, and S. M. Reppert, Eds., pp. 405-418, Oxford University Press, New York, NY, USA, 1991.

[3] D. S. Carter and B. D. Goldman, "Antigonadal effects of timed melatonin infusion in pinealectomized male Djungarian hamsters (Phodopus sungorus sungorus): duration is the critical parameter," Endocrinology, vol. 113, no. 4, pp. 1261-1267, 1983.
[4] E. L. Bittman and F. J. Karsch, "Melatonin and photorefractoriness: loss of response to the melatonin signal leads to seasonal reproductive transitions in the ewe," Biology of Reproduction, vol. 30, no. 2, pp. 585-593, 1984.

[5] H. Underwood and B. D. Goldman, "Vertebrate circadian and photoperiodic systems: role of the pineal gland and melatonin," Journal of Biological Rhythms, vol. 2, no. 4, pp. 279-315, 1987.

[6] V. M. Cassone, "Effects of melatonin on vertebrate circadian systems," Trends in Neurosciences, vol. 13, no. 11, pp. 457-464, 1990.

[7] D. L. Krause and M. L. Dubocovich, "Melatonin receptors," Annual Review of Pharmacology and Toxicology, vol. 31, pp. 549$568,1991$.

[8] B. Stankov, F. Fraschini, and R. J. Reiter, "Melatonin binding sites in the central nervous system," Brain Research Reviews, vol. 16, no. 3, pp. 245-256, 1991.

[9] M. Karasek and K. Winczyk, "Melatonin in humans," Journal of Physiology and Pharmacology, vol. 57, supplement 5, pp. 19-39, 2006.

[10] K. Petrie, J. V. Conaglen, L. Thompson, and K. Chamberlain, "Effect of melatonin on jet lag after long haul flights," British Medical Journal, vol. 298, no. 6675, pp. 707-710, 1989.

[11] T. A. Wehr, "The durations of human melatonin secretion and sleep respond to changes in daylength (photoperiod)," Journal of Clinical Endocrinology and Metabolism, vol. 73, no. 6, pp. 1276-1280, 1991.

[12] P. Delagrange and B. Guardiola-Lemaitre, "Melatonin, its receptors, and relationships with biological rhythm disorders," Clinical Neuropharmacology, vol. 20, no. 6, pp. 482-510, 1997.

[13] L. Wetterberg, J. Beck-Friis, and B. F. Kielmann, Biological Rhythms, Mood Disorders, Light Therapy and the Pineal Gland, AmericanPsychiatric, Washington, DC, USA, 1990.

[14] M. Karasek, R. J. Reiter, D. P. Cardinali, and M. Pawlikowski, "The future of melatonin as a therapeutic agent," Neuroendocrinology Letters, vol. 23, supplement 1, pp. 118-121, 2002.

[15] F. A. Al-Omary, “Melatonin: comprehensive profile," Profiles of Drug Substances and Excipients Related Methodology, vol. 38, pp. 159-226, 2013.

[16] M. Somei, Y. Fukui, M. Hasegawa, N. Oshikiri, and T. Hayashi, "Syntheses of melatonin and its derivatives," Heterocycles, vol. 53, no. 8, pp. 1725-1736, 2000.

[17] A. Mishra, R. Veerasamy, P. K. Jain, V. K. Dixit, and R. K. Agrawal, "Synthesis, characterization and pharmacological evaluation of amide prodrugs of ketorolac," European Journal of Medicinal Chemistry, vol. 43, no. 11, pp. 2464-2472, 2008.

[18] N. H. Nam, S. Sardari, M. Selecky, and K. Parang, "Carboxylic acid and phosphate ester derivatives of fluconazole: synthesis and antifungal activities," Bioorganic and Medicinal Chemistry, vol. 12, no. 23, pp. 6255-6269, 2004.

[19] N. H. Nam, Y. Kim, Y. J. You, D. H. Hong, H. M. Kim, and B. Z. Ahn, "Water soluble prodrugs of the antitumor agent 3-[(3-amino-4-methoxy)phenyl]-2-(3,4,5-trimethoxyphenyl)cyclopent-2-ene-1-one," Bioorganic and Medicinal Chemistry, vol. 11, no. 6, pp. 1021-1029, 2003.

[20] S. Penugonda, A. Kumar, K. Agarwal, K. Parang, and R. Mehvar, "Synthesis and in vitro characterization of novel dextran-methylprednisolone conjugates with peptide linkers: effects of linker length on hydrolytic and enzymatic release of methylprednisolone and its peptidyl intermediates," Journal of Pharmaceutical Sciences, vol. 97, no. 7, pp. 2649-2664, 2008. 

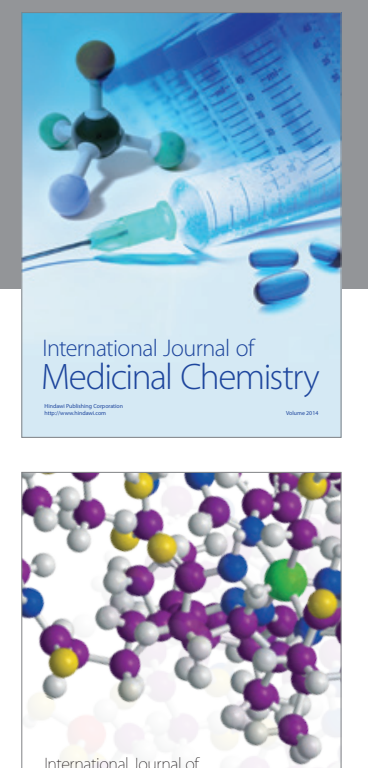

\section{Carbohydrate} Chemistry

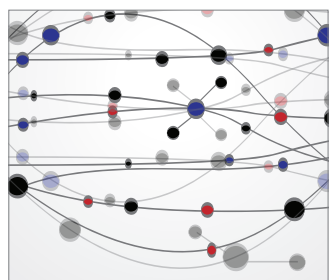

The Scientific World Journal
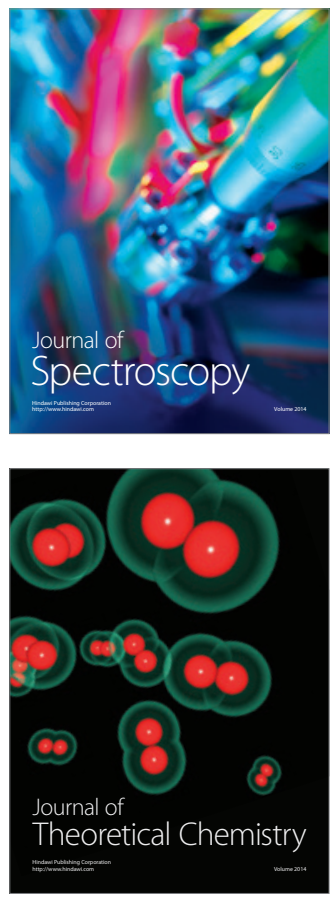
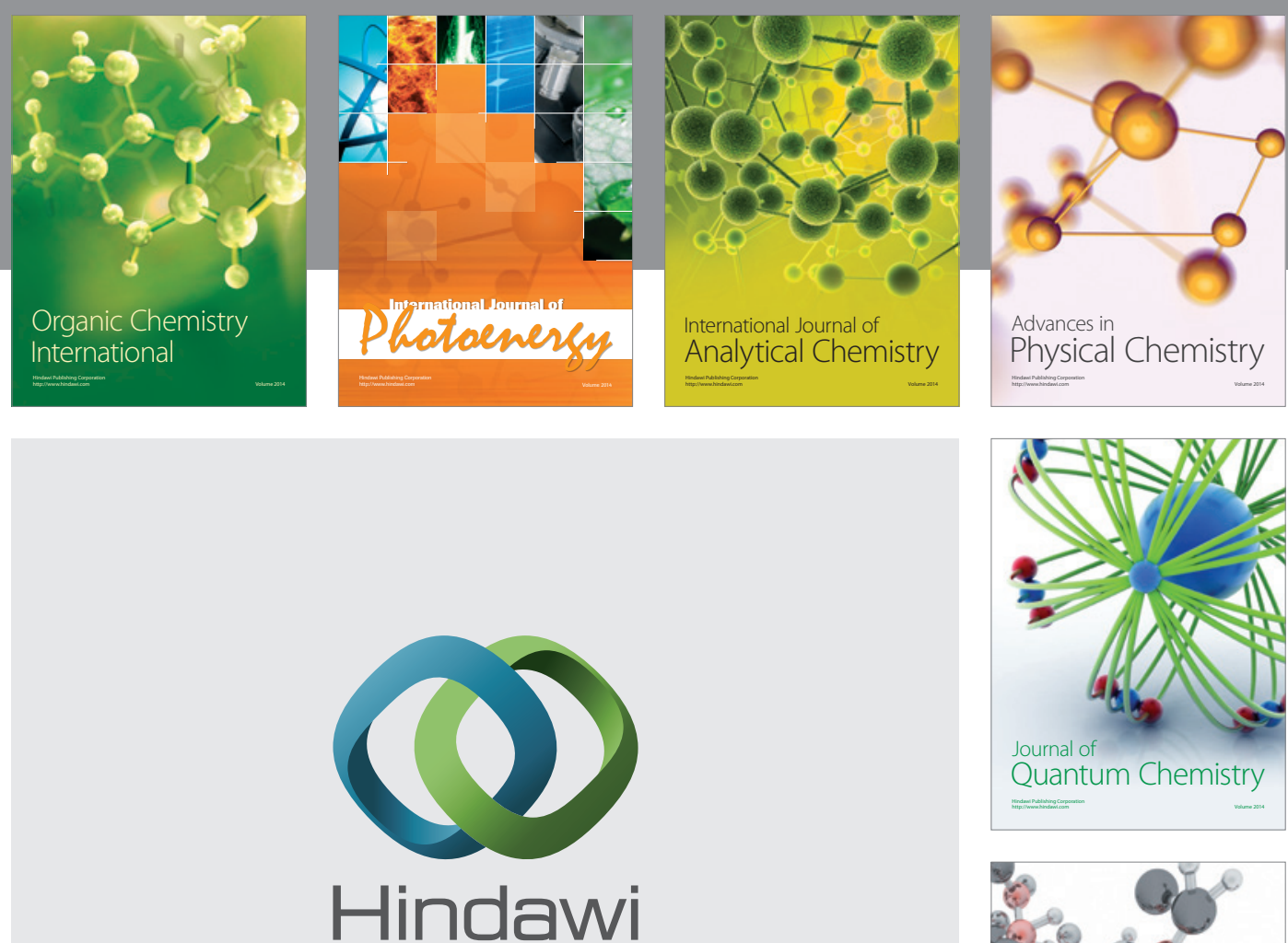

Submit your manuscripts at

http://www.hindawi.com

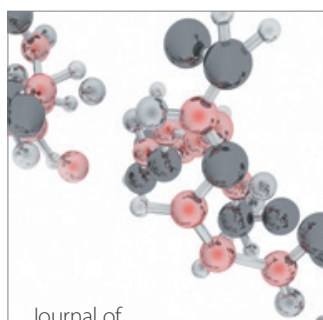

Analytical Methods

in Chemistry

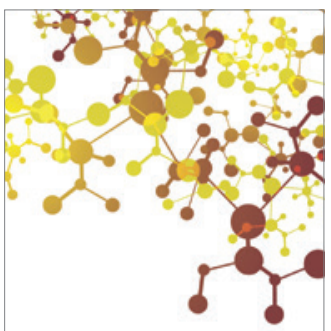

Journal of

Applied Chemistry

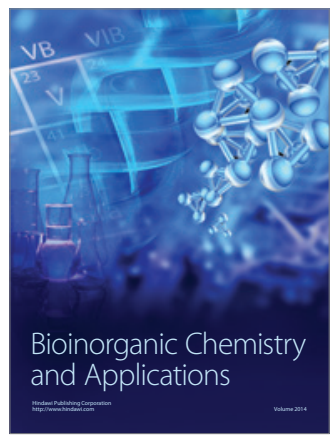

Inorganic Chemistry
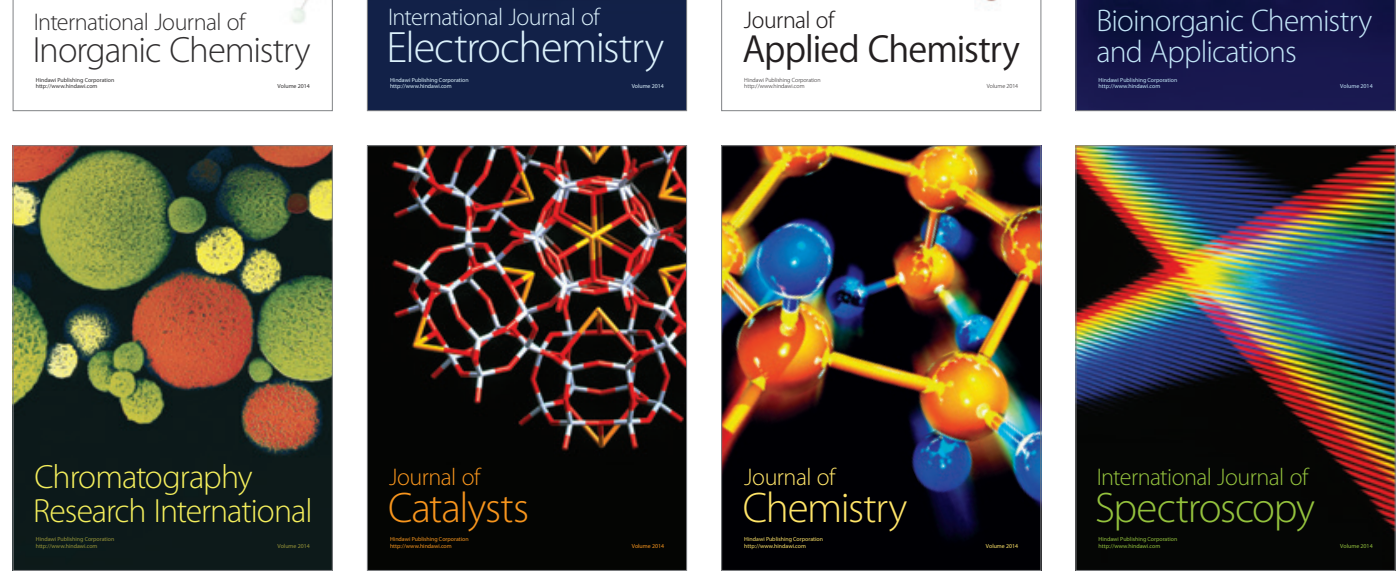